\title{
Vesiculobollous disease with Cutaneous Systemic Lupus Erythematosus Treated by Rituxima; a case report
}

\author{
Wigdan Mohammed Niamat Alla ${ }^{1}$, Burhan Mohamed Ali Ahmed ${ }^{1}$, Mohammed Elmujtba \\ Adam Essa ${ }^{2}$, Atif Elhadi Abdalla Babker ${ }^{3}$, and Elnour Mohammed Elagib ${ }^{1}$ \\ ${ }^{1}$ Sudan Medical Specialization Board \\ ${ }^{2} \mathrm{Al}$ Fashir University Faculty of Medicine \\ ${ }^{3}$ University of Gezira
}

October 1, 2021

\begin{abstract}
SLE is a chronic autoimmune disease characterized by multisystem inflammation, Vesiculobullous (VB) are different groups of oral disorders characterized by the formation of bullae. The aim of this report is to describe a case of a vesiculobullous disease in SLE.
\end{abstract}

\section{Introduction}

SLE is an autoimmune condition with a variable range of clinical features from skin disease to systemic involvement $(1,2)$. VB diseases are a distinct group of oral disorders characterized by the formation of vesicles or bullae (3). bullous systemic lupus erythematosus (BSLE), is a rare blistering condition that mainly happens in females (4), the incidence of SLE is about 1 to 10/100,000 annually, and his prevalence around 6-130/100,000 (5). Approximately 60-85\% of SLE patients have cutaneous involvement, however, near $5 \%$ may develop vesiculobullous lesions, which occur in the form of bullae, vesicles, erosions and sometimes crusts (6).

BSLE is marked by the rapid and widespread progress of tense bullae and vesicles over erythematous macules or plaques. Preferential sites are: proximal superior limbs, superior trunk and the face. Mucosal involvement is common in the laryngeal, pharyngeal, perioral, and genital areas. The lesions usually develop with no scarring, but hyper or hypochromia can occur (7). Here we believed that this case report will receive the most attention from Sudanese clinicians and rekindle knowledge and awareness in regard to vesiculobollous disease in patient who is known to be suffering from SLE.

\section{Case report}

A 43-year-old Sudanese housewife, presented on June 2021 at Omdurman military hospital, Khartoum, Sudan. With recurrent painless oral ulceration (Fig 1) for the last 2 years associated with joints pain affecting the metacarpophalangeal joints (MCP), proximal interphalangeal joints (PIP), wrist joint of both hands, morning stiffness lasting for more than an hour and generalized fatigue. Although, she has no skin rash, genital ulcers, muscle pain, dry eye or mouth. In September 2020, she was diagnosed with SLE based on her clinical presentation and the positive finding of ANA profile which was strongly positive for Ribonucleoproteins Abs (RNP), anti-Smith Abs(Sm), anti-Ribosomal Proteins Abs (Ribp) and microalbuminuria. For which, Prednisolone 20mg, hydroxychloroquine (HCQ) $200 \mathrm{mg}$ and azathioprine 50mg were prescribed. Two months later after the oral ulceration minimally improved new lesions in form of macular rash with vesicle and blister start to appear in her mouth, face, trunks, upper and lower limbs (fig 2). When the blister is 
ruptured they lifted a hyper and hypopigmentation mark without scarring. During this period she lost considerable weight and has a fever, hair loss and headache. The patient has been referred to the dermatology department, in which a blood test for vitamin b12 level and desmoglein 1 and 3 were, ordered and their result was normal, therefore, topical treatments were prescribed without a clear diagnosis. After five months the patient developed left side weakness of sudden onset. Although, her Cardiovascular, respiratory, Gastrointestinal, genitourinary systems were unremarkable. She has no history of any neurological disease, diabetes or hypertension. She has a family history of SLE related to her sister. Her Clinical examination upon admission showed generalized thin-walled bullae, some of them are hemorrhagic with erythematous macule and area of hyper and hypopigmentation, however, no evidence of active synovitis was detected. Central nervous system examination showed no sign of meningism or cranial nerve involvement, she was conscious, oriented with GCS 15/15. The left side of her body revealed an increased tone and reflex, power grade three with an upgoing plantar reflex, and a normal right side. Full workup was done showed low hemoglobin, high erythrocyte sedimentation rate (ESR) and C reactive protein 13, Normal urine analysis without active sediment, renal profile, liver profile, Vitamin B12 level, Antiphospholipid antibodies, chest x-ray, echocardiography, electrocardiograph, viral screening and lipid profile were all normal. Antinuclear Antibody (ANA) profile showed strong positive ( $\mathrm{RNP} / \mathrm{Sm}$ ab, $\mathrm{Sm}$ ab, anti- RibP ab), urine for albumin/creatinine ratio was $9.6 \mathrm{mg} / \mathrm{mmol}$ (microalbuminurea), C3 and C4 were low. Desmoglien1 Abs is 16, desmoglien 3 Abs is 18. Based on the clinical and laboratory investigation a diagnosis of vesiculobullous disease with SLE was established. The patient received Hydrocortisone intravenously (IV) 100mg, Pantoprazole $40 \mathrm{mg}$ IV, Paracetamol $1 \mathrm{~g}$ iv along with Mebo cream, fusederm cream and B.protien powder, Metronidazole 500mg iv, Ceftriaxone $1 \mathrm{gm}$, flucloxacillin and Tonics and IVIM $4 \mathrm{mg} / \mathrm{KG}$ /day for three days. Her condition was improved partially However, bullous formation still continues to appear, hence, rituximab 500mg/weekly for 4 weeks has been administrated followed by mycophenolate mofetil $500 \mathrm{mg}$ BD. A few days later the patient discharge with a marked response to the treatment (Fig 3). Now she is on regular follow up.

\section{Discussion}

A vesiculobullous disease is a rare autoimmune blistering disease that commonly occurs as tense bullae with or without vesicles in patients with SLE (8). Due to the rarity of the condition, his epidemiology data are restricted, however, some reports showed an Incidence of 3.4/million/year. The incidence of this disease with SLE is about 1\%. As the knowledge about it is mainly based on case series or reports $(7,9)$. Patients with the vesiculobullous disease usually present with clinical features of SLE, the blisters mainly occur on the oral mucosa, neck, face and upper extremities (10). All the reported cases indicated these bullae were mostly triggered by sun exposure, they were also tense and heal with no scarring (8). This is a classical presentation of our reported case as it's already diagnosed with SLE, the bullae occurred on the mouth, face, neck and extremities. It was tense and was healed without any scars behind.

The pathophysiology of VB is mostly multifactorial, which includes environmental triggers, genetic predisposition and ultraviolet aggravation leading to adaptive and innate immune response (11). Activation of the immune system leads to the activation of self-antigen-specific $\mathrm{T}$ cells, autoreactive cytotoxic $\mathrm{T}$ lymphocytes from dermal autoantibody deposition cause hydropic degeneration of the basal-cell layer of the epidermis and apoptotic keratinocytes (12). The diagnosis of VB diseases should be done according to immunological, histopathological and clinical grounds. Mucosal disorder and bullae can be diagnosed from clinical examination and brief history. The histopathology commonly shows sub-epidermal bullae with neutrophilpredominant inflammatory infiltrate below the bullae formation. Also, microabscesses can be present in the dermal papillae is also frequent (13). Unfortunately, our patient refused to undergo though the tissue biopsy procedure, so the diagnosis was mainly based on Immunological assay and clinical ground. Immunological assay for VB mainly can be done by detecting high desmoglien antibodies (DSG), DSG2 was found in all desmosome-possessing tissues, such as nonepithelial myocardium, however, the expression of DSG1 and DSG3 was mainly limited to the epidermis and mucosa (14). DSG delivered insight into the pathophysiology of a more common disease, bullous as well as the associated disease (15). In our patient, both DSG1 and DSG2 were significant to the diagnosis. The erosions and blisters are formed by circulating immunoglobulin (IgG) autoantibodies against DSG1 and DSG3, adhesion molecules in desmosomes that have an essential role 
in the cohesion between keratinocytes (16). The first treatment of autoimmune bullous diseases is systemic corticosteroids in combination with other immunosuppressants drugs to decrease autoantibody production (17), In most patients, this course of treatment is effective, however, in refectory cases more targeted drugs against autoantibody production such as rituximab are used (18).

In conclusion, a middle-aged Sudanese woman with SLE, presented with painless oral ulcer and joint pain for the last two years during the treatment she developed vesicles and blisters in most of her body part, Clinical and immunological investigation revealed a diagnosis of VB associated with SLE, the patient received regiment treatment of steroids but her condition not improved, then rituximab has been used, which showed good response.

\section{Acknowledgments}

The case was diagnosed and treated in Dr Elnour Mohammed Elagib rheumatology unite at Omdurman Military hospital, Khartoum, Sudan.

\section{Ethical approval and consent to publish}

Obtained

\section{Conflict of Interest}

Non

\section{Written consent from the patient}

Obtained

\section{Funding}

No fund have been received

\section{Authors' contribution}

All authors contributed equally

\section{Availability of data and materials}

All the data used in the study are available from the first and corresponding author on reasonable request.

\section{References}

1. Manson JJ, Rahman A. Systemic lupus erythematosus. Orphanet J Rare Dis. 2006 Mar 27;1:6. PubMed PMID: 16722594. Pubmed Central PMCID: PMC1459118.

2. Cojocaru M, Cojocaru IM, Silosi I, Vrabie CD. Manifestations of systemic lupus erythematosus. Maedica (Bucur). 2011 Oct;6(4):330-6. PubMed PMID: 22879850. Pubmed Central PMCID: PMC3391953.

3. Krupaa J, Balachander N, Janani S, Sankari SL. Diagnosis of Vesicullo Bullous Lesions-Simplified. Biomedical and Pharmacology Journal. 2016;9(3):1063-6.

4. Nico MM, Lourenco SV. Multiple blisters along the lip vermilion are a clue to bullous lupus erythematosus. Acta Derm Venereol. 2012 Jul;92(4):404-5. PubMed PMID: 22293956.

5. Pons-Estel GJ, Salerni GE, Serrano RM, Gomez-Puerta JA, Plasin MA, Aldasoro E, et al. Therapeutic plasma exchange for the management of refractory systemic autoimmune diseases: report of 31 cases and review of the literature. Autoimmun Rev. 2011 Sep;10(11):679-84. PubMed PMID: 21569864.

6. Contestable JJ, Edhegard KD, Meyerle JH. Bullous systemic lupus erythematosus: a review and update to diagnosis and treatment. American journal of clinical dermatology. 2014 Dec;15(6):517-24. PubMed PMID: 25358414. 
7. Padrao EMH, Teixeira LF, Maruta CW, Aoki V, Felipe da Silva AS, Kim EIM, et al. Bullous systemic lupus erythematosus - a case report. Autopsy \& case reports. 2019 Jan-Mar;9(1):e2018069. PubMed PMID: 30863736. Pubmed Central PMCID: 6394362.

8. Torres Saavedra FA, Campo LR, Mendez MV, Barreneche NM, Suaza GAV, Restrepo JDR, et al. Bullous lupus as the first manifestation of systemic lupus erythematosus in the pediatric population: A diagnostic challenge in daily practice. Lupus. 2020 Dec;29(14):1937-42. PubMed PMID: 32842868.

9. Elqatni M, Qacif H, Zyani M, Amezyane T, Ghafir D. Bullous systemic lupus erythematosus. Presse medicale. 2018 Feb;47(2):190-1. PubMed PMID: 29478791.

10. Lourenco DM, Gomes RC, Aikawa NE, Campos LM, Romiti R, Silva CA. Childhood-onset bullous systemic lupus erythematosus. Lupus. 2014 Nov;23(13):1422-5. PubMed PMID: 25074872.

11. Hejazi EZ, Werth VP. Cutaneous Lupus Erythematosus: An Update on Pathogenesis, Diagnosis and Treatment. American journal of clinical dermatology. 2016 Apr;17(2):135-46. PubMed PMID: 26872954.

12. Achtman JC, Werth VP. Pathophysiology of cutaneous lupus erythematosus. Arthritis Res Ther. 2015 Aug 10;17:182. PubMed PMID: 26257198. Pubmed Central PMCID: 4530484.

13. Lewis D, Logas DB, Wojciechowski J, Friberg C. Bullous eruption in a patient with systemic lupus erythematosus. Journal of the American Academy of Dermatology. 1998 Jun;38(6 Pt 1):1013-4. PubMed PMID: 9632025.

14. Schafer S, Koch PJ, Franke WW. Identification of the ubiquitous human desmoglein, Dsg2, and the expression catalogue of the desmoglein subfamily of desmosomal cadherins. Experimental cell research. 1994 Apr;211(2):391-9. PubMed PMID: 8143788.

15. Stanley JR, Amagai M. Pemphigus, bullous impetigo, and the staphylococcal scalded-skin syndrome. The New England journal of medicine. 2006 Oct 26;355(17):1800-10. PubMed PMID: 17065642.

16. Kurihara Y, Yamagami J, Funakoshi T, Ishii M, Miyamoto J, Fujio Y, et al. Rituximab therapy for refractory autoimmune bullous diseases: A multicenter, open-label, single-arm, phase $1 / 2$ study on 10 Japanese patients. The Journal of dermatology. 2019 Feb;46(2):124-30. PubMed PMID: 30585649.

17. Fardet L, Flahault A, Kettaneh A, Tiev KP, Genereau T, Toledano C, et al. Corticosteroid-induced clinical adverse events: frequency, risk factors and patient's opinion. The British journal of dermatology. 2007 Jul;157(1):142-8. PubMed PMID: 17501951.

18. Eisenberg R, Looney RJ. The therapeutic potential of anti-CD20 "what do B-cells do?". Clinical immunology. 2005 Dec;117(3):207-13. PubMed PMID: 16169773.

Figure (1): showed the oral ulcer 


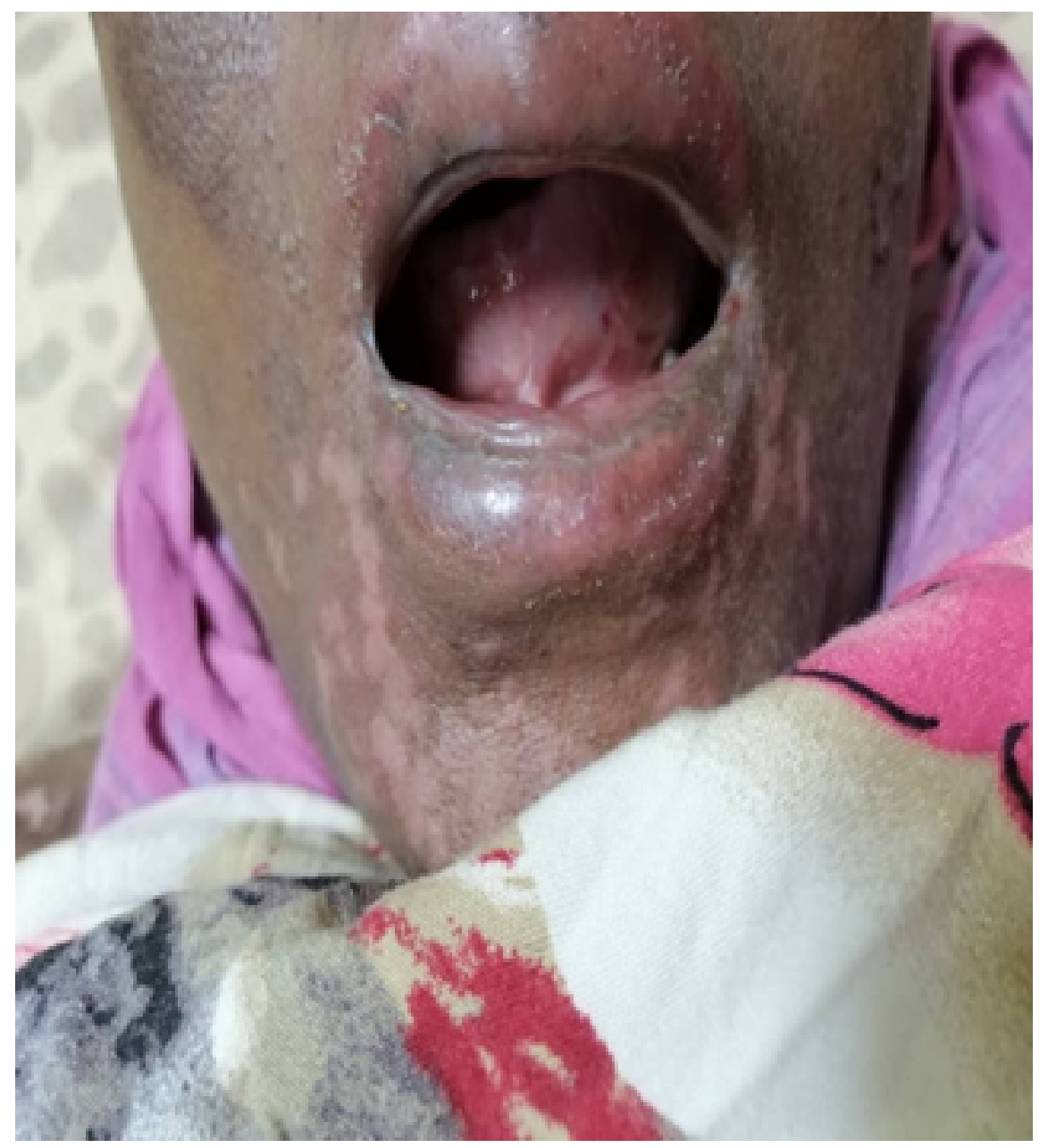

Figure (2): Showed tense bullae over erythematous macules 


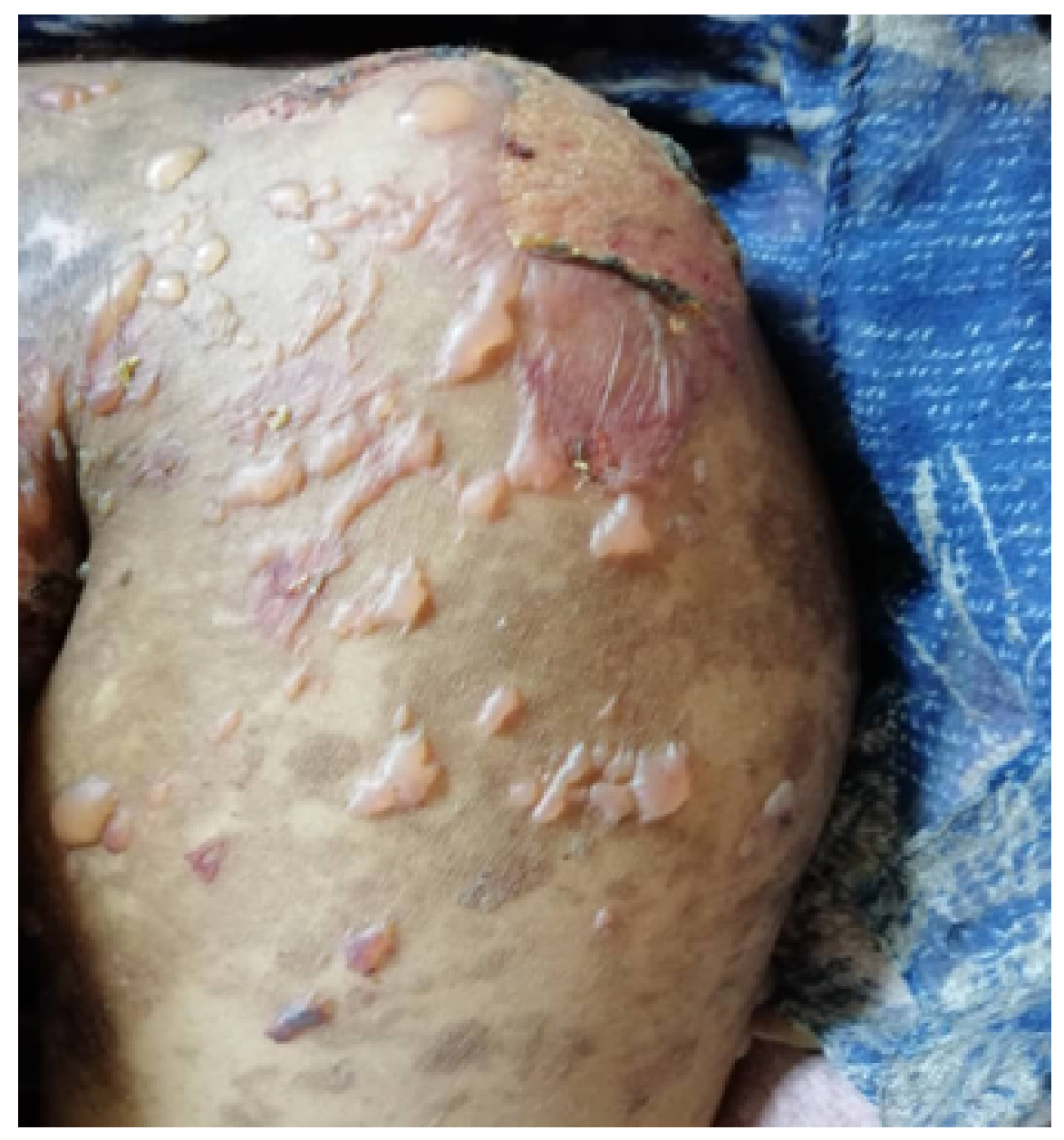

Figure (2): showed linear tense bullae of varying sizes over erythematous macules and plaques, A: showed the bullae before treatment, B: during treatment, C: after rituximab administration.

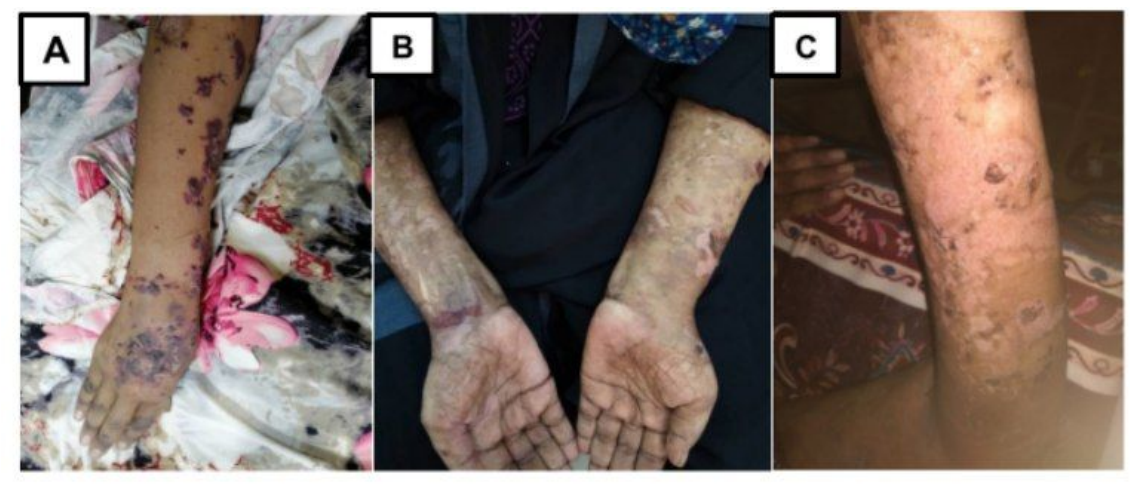

